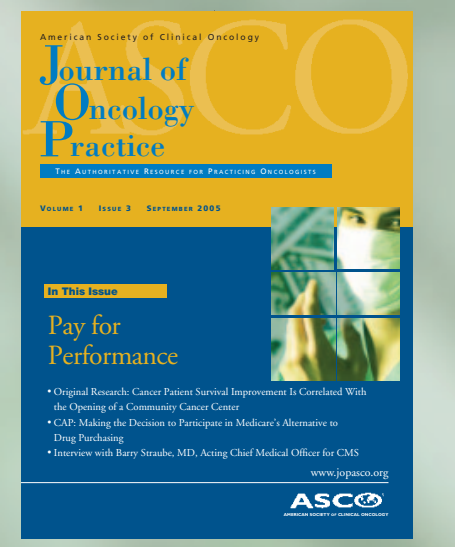

\title{
Pay for Performance
}

\section{In Cancer Care, Now Is the Era of Defining, Measuring, and Paying for Quality}

he era of quality measurements in cancer care has arrived, and close behind it, many believe, is a future of reimbursement linked to quality outcomes-pay for performance. Initiatives that could impact every oncologist in practice are underway. At the national level, under a leadership committed to driving the adoption of paying for performance and paying for quality, the Centers for Medicare and Medicaid Services (CMS) has several quality cancer care projects and demonstrations in progress or planned. The National Quality Forum (NQF) is developing national consensus quality measures for oncology, which could be adopted for use by CMS for its oncology programs.

At the same time, many practicing oncologists throughout the country are taking steps to assess and improve the quality of care they provide. One emerging program is the Quality Oncology Practice Initiative (QOPI), a voluntary auditfeedback program to assist oncologists in quality improvement. Launched 2 years ago and soon to roll-out nationally, QOPI is designed to assess a number of indicators of quality oncology care within an office or practice setting, and provide reports that identify areas of strength and those in need of improvement. Its ultimate goal is to encourage and help practices to develop a culture of self-examination and improvement-a culture change that will, among other benefits, help prepare oncologists for the pay-for-performance paradigm.

\section{CMS Perspective}

Speaking at the ASCO 2005 Annual Meeting, Barry M. Straube, MD, acting director of the Office of Clinical Standards and Quality at CMS, underscored the broad ambitions of his office to impact the entire health care landscape. As he explained, "One of the key changes that has occurred over the past year is that Mark McClellan, administrator of CMS, has been speaking on a national level about the concept of CMS as a public health agency in the sense of using the influence and the revenue strength that CMS has to transform the U.S. health care system across all payer systems." Toward this end, a number of quality initiatives are already in place and others are in the planning stages. All, according to Straube, are incorporating necessary aspects of pay for performance. They include initiatives targeting nursing homes, home health organizations, hospitals, and increasingly, physicians' offices.

"Pay for performance and paying for quality is something that CMS will be driving nationally, and already has started to do." Barry M. Straube, MD, CMS acting director, Office of Clinical Standards and Quality

Many practicing oncologists are familiar with the Quality Cancer Care Demonstration, which aims to address improved quality of care for patients undergoing chemotherapy. CMS announced the project on November 1,2004, as a 1-year demonstration with an investment of $\$ 300$ million.

According to CMS, specific goals are to reduce overall costs by avoiding hospitalizations due to complications, in part by managing pain, minimizing nausea and vomiting, and limiting fatigue.

Standardized assessment scales for measurement of pain, nausea and vomiting, and fatigue have been incorporated into the CMS billing codes (Table 1). By billing CMS using the designated codes, practitioners self-enroll in the program. Practices reporting on all three factors qualify for an additional payment.

Other relevant demonstration projects include

- the Medicare Health Care Quality Demonstration Programs (Section 646 of MMA). Expanded from a demonstration started a year and a half ago, the program is designed to ascertain whether economies of scale can deliver savings in health care settings just as they often do in large corporations. The program involves a number of large physician group practices (250 physicians or more) in a distinct pay-for-performance scheme. Straube explained, "Individuals are reimbursed [by 
calculating] the actuarial projection of what a risk-adjusted cadre of patients would normally consume under the Medicare program. If efficiencies and economy of scale in these large physician groups result in care that is delivered for less than the traditional fee-for-service programs, [participants] share in up to $90 \%$ of the savings over the baseline of what Medicare fee-for-service would normally provide."

- a quality cancer demonstration for ethnic and racial minorities. The goals of the demonstration are to reduce disparities in care, improve early detection and treatment, and help beneficiaries navigate the health care system in a timely and informed manner. Target populations are American Indians, Asian Americans and Pacific Islanders, African Americans, and Latino individuals. Cancer targets are breast, cervix, colon/rectal, prostate, and lung. This program is designed as a capitated model, Straube explained, “...capitating only those individuals who wish to participate either with the prevention wing/limb, or the treatment of cancer limb."

Results from these demonstrations will likely be applied in the near term to broader pay-for-performance models. As Straube concluded, "We are trying to take the methods in the demonstration projects and various other initiatives to begin dialogues with professional societies, including ASCO, to see if we can come up with ways that we can implement pay-forperformance programs using some of the quality metrics we've described, over the next year or two."

\section{National Standards for Quality Measurement: The Role of the National Quality Forum}

The National Quality Forum (NQF) is a private, not-forprofit voluntary consensus standards-setting organization. Initiated in 2000, NQF is an "organization of organizations" with more than 260 member groups and member councils that represent all major stakeholders-consumers, health care providers and health plans, purchasers, and research and quality improvement organizations - to represent diverse perspectives on quality.

Rodger J. Winn, MD, is director of the Cancer Project for the National Quality Forum. In an address at the 2005 ASCO Annual Meeting, Winn picked up on the pay-forperformance theme by discussing the need for vetted, standard quality measures - especially as the data collected are used in increasingly consequential ways. For instance, although the concept of public reporting is uncommon in oncology, it is quite familiar to cardiac surgeons, as Winn explained: "If you live in New York or California, your 30day mortalities for cardiac surgery are reported in public venues, and consumers can access this information and base their choice of doctors on these data." Public reporting in turn drives selection of physicians, and that leads to pay for performance "...as a natural outcome of this cascade.

An additional use for measurement outcomes is for national surveillance and allocation of national resources. A third major use for measures is internal quality improvement, which involves comparing performance to benchmarks, and then instituting office-based or practice-based changes that will bring about improvement.

Public reporting of quality measures drives selection of physicians and pay for performance follows as a natural outcome of this cascade.

NQF undertakes its mission of measure standardization in an environment with multiple cancer measures and guidelines.

Being noncentralized, the measures are sometimes fragmented and can be duplicative or even contradictory, thus adding to confusion and difficulties when gathering data.

To begin to address this problem, the steering committee for the NQF Cancer Project first met in September of 2002. The group identified priority areas based on a set of criteria that included consistency with national goals, importance, and known disparities or serious quality issues. Disease-specific priorities were identified as breast, colorectal, and prostate cancers. Cross-cutting priority areas were identified as access and competence; communication/coordination/IT; prevention and screening; and symptom management and end-of-life care.

A second phase of the NQF cancer project began in May of 2004, focusing on endorsing a set of voluntary consensus standards for quality of cancer care for use by the public and private sectors. The priority areas were further refined to breast cancer diagnosis and treatment, colorectal cancer diagnosis and treatment, and symptom management and end-

Table 1. Quality Cancer Care Demonstration G-codes

\begin{tabular}{|l|c|c|c|c|}
\hline Factor & $\begin{array}{c}\text { “Not at all” } \\
\text { Level 1 }\end{array}$ & $\begin{array}{c}\text { “A Little” } \\
\text { Level 2 }\end{array}$ & $\begin{array}{c}\text { “Quite a Bit” } \\
\text { Level 3 }\end{array}$ & $\begin{array}{c}\text { "Very Much” } \\
\text { Level 4 }\end{array}$ \\
\hline Nausea and/or vomiting & G9021 & G9022 & G9023 & G9024 \\
\hline Pain & G9025 & G9026 & G9027 & G9028 \\
\hline Lack of energy & G9029 & G9030 & G9031 & G9032 \\
\hline
\end{tabular}




\section{PAY FOR PERFORMANCE IN LARGE CLINICAL PRACTICES}

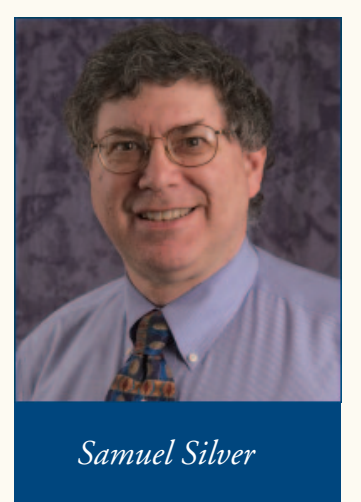

In the spring of 2005, CMS selected 10 large clinical sites around the United States (200 or more physicians) to participate in its 3-year demonstration project as a test of the pay-for-performance concept in Medicare's fee-forservice payment system. Participants are challenged to find ways to decrease the cost of care for patients enrolled in Medicare Parts A and B, and are incentivized by a return of a percentage of the costs savings from CMS. A second part of the project involves adherence to quality outcome measures in coronary artery disease, congestive heart failure, diabetes, prevention issues such as vaccinations and colorectal screening, and blood pressure measurements. The "earn back" is based on a combination of cost savings and adherence to quality measures according to a progressive schedule of cost/quality weighting (70/30 in year $1 ; 60 / 40$ in year 2 ; and $50 / 50$ in year 3$)$. The University of Michigan Faculty Practice Group is one of the sites participating in this demonstration, and Samuel Silver, MD, PhD, professor of internal medicine/ hematology-oncology at the University of Michigan is a member of the university's CMS demonstration project design team.

This is a new program for CMS and the participants, but Silver and his colleagues have by now had the opportunity to examine the program from the inside, and to highlight some of its challenges. "It is not totally clear to us that we know exactly what these quality outcome points are and exactly how CMS wants us to measure them. This is still being debated within the $U$ of $M$ practice group, as is whether it is appropriate that CMS tell us how they want to [select and evaluate the measures] as opposed to how the big centers want to do them."

Another issue is the cost to the institutions and centers taking part. "There are huge amounts of infrastructure costs in trying to get one of these programs together. And it is not clear to anyone, I don't think, whether the incentives you will potentially receive will actually pay for the infrastructure that must be put into the program," Dr. Silver said. This is not to say that the clinical centers do not themselves want to institute quality measures and identify areas for potential cost savings. "The bottom line is that we [University of Michigan] thought we should be doing this anyway-instituting efficiencies [incorporating quality outcome measures] with or without the CMS demonstration project. It's the right thing to do," he said, "but we just don't think CMS realizes that this may cost more than the incentives that may come back [to the practice]."

At the University of Michigan, a few things have been learned about the patients who cost Medicare the most. According to Silver, they are patients with coronary artery disease or diabetes, or heart failure who require hospital re-admissions. One of the things that the group at the university is looking at is transitional care between discharge and their first visit with their primary caregiver. "If we could make things better, we will probably decrease the number of re-admissions and decrease the overall cost to Medicare. And, as we decrease the amount of overall costs for the care of these Medicare beneficiaries up to a certain limit, the more we can get in terms of incentive payments."

of-life care. Technical panels have been convened in each priority area to review candidate measures and make specific recommendations. For example, the breast cancer technical panel reviewed 25 submitted measures and accepted five for quality improvement. The five measures being forwarded to the steering committee are

- axillary lymph node dissection and sentinel node biopsy in early stage breast cancer

- needle biopsy prior to surgery

- post-lumpectomy radiotherapy
- combination chemotherapy in estrogen receptor-negative patients

- percentage of breast cancer-conserving surgery

Member council and board reviews of measures for these priority areas are underway; approvals are projected for May through July of 2006, with the final report, including research recommendations, in September of 2006.

Through these and other coordinated efforts, the NQF is poised to serve as the final conduit for the endorsement of a national cancer measure set. 


\section{Quality Oncology Practice Initiative}

By contrast with these national initiatives, QOPI aims to help oncologists improve care in their own offices. The initiative began when Joseph Simone, MD, of Simone Consulting, partnered with ASCO to assemble a group of willing oncologists and create the basic QOPI framework, consisting of quality indicators, a chart abstraction tool, and a process defining how practices might participate. Data collection began 2 years ago.

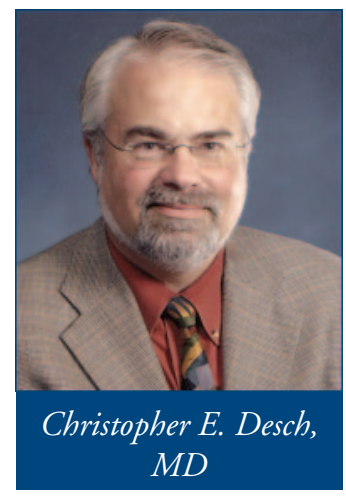

A critical element of the system is the QOPI measures. The measures were developed by the participating oncologists and are drawn from several spheres: consensus-based, clinically relevant components such as flow sheets, pathology reports, staging and informed consent; concordance with practice guidelines such as the use of anti-emetics, growth factors, and tumor markers; standards of care such as for pain control; and measures tested and validated by the National Initiative on Cancer Care

Quality (NICCQ). "Repeated measurements are the foundation for improvement," said Chris Desch, MD, who practices at the Virginia Cancer Institute and is one of the founders of QOPI. "They may not be sufficient for improvement, but they are necessary in order to provide direction for improvement efforts. In addition, the efforts have to result in benefit to both the practice and the patients."

Each physician participating in QOPI oversees data collection in his immediate office setting. Oncologists practicing in multi-office practices can choose to collect data at several offices, and each practice has the option of submitting data at the individual physician level. Each data collection requires 75 to 85 charts. The abstractors, who are trained by ASCO staff, are clinical or clerical office staff. Data are entered directly onto a secure ASCO Web form at intervals of 6 months.

Since QOPI's inception, more than 4,000 charts have been abstracted in 23 practices. Most of the practices have submitted data two or more times. Following each round of data collection, participating practices receive a quality report that displays the performance of the practice in contrast to the QOPI average.

Michael Neuss, MD, a QOPI founder from Oncology Hematology Care Inc. in Cincinnati, Ohio, commented on the value of the program to his practice: "QOPI is a wonderful tool to help examine and improve our practice. It provides us with a group of quantitative measures that can be scored and thereby allows us to see how we are doing relative to other practices. Perhaps more importantly, it provides us with a community of practices with whom we can share ideas - about what the important components of high-quality care are, how to achieve [this level of care] and how these components may relate to payer bonuses when and if the pay for performance concept is actually realized." In their QOPI data report, Neuss and colleagues discovered that although the practice was doing well in general, they need to pay more attention to pain control in dying patients - or better document the care they are providing.

QOPI data collected thus far amplify areas where participating oncology practices uniformly perform to the highest standards, but also areas where they differ by wide margins. For example, chemotherapy was recommended for breast cancer patients meeting NICCQ criteria in nearly $100 \%$ of cases. On the other hand, in only about one third of practices had all patients signed a consent form before chemotherapy administration. In about $25 \%$ of responses, treatment was not discussed and patient consent not documented in the chart.

\section{Participating in QOPI will meet the ABIM performance improvement (part IV) requirements for maintenance of certification. (Part IV will be mandatory for oncologists as of January 1, 2006.)}

Addressing pain at the final visit before death was another area where improvements could be made in many practices. Among the QOPI practices, pain was assessed on the visit before death only $65 \%$ of the time. An additional quality indicator is that pain should be rated by number or degree. This is done infrequently in many practices, according to the QOPI findings.

Any quality improvement system has its limitations, Desch admitted, but he noted that QOPI offers a number of both negative and positive incentives. "It is absolutely free when Anthem rates your practice or when Cigna rates your practice, but I suggest that oncology practices may wish to do this for themselves first, using this methodology and these measures."

Ultimately, the goal of QOPI is not to provide a report card, according to Simone, "but to help [a practice] develop a culture of self-examination." The QOPI process of collecting data may be an important step in the process of adapting to pay for performance. An immediately tangible benefit, Simone added, is for oncologists that require American Board of Internal Medicine (ABIM) re-certification. Participating in QOPI will meet the ABIM performance improvement (part IV) requirements for maintenance of certification. (Part IV will be mandatory for oncologists as of January 1, 2006.) A national roll-out of QOPI is planned by the end of 2005, allowing interested oncologists to join the initiative.

For questions about QOPI and QOPI enrollment, contact Kristen McNiff, MPH, Assistant Director, Cancer Care Quality, Cancer Policy and Clinical Affairs, (qopi@asco.org). 